\title{
EL SUICIDIO EN EL ÁMBITO COMUNITARIO: LINEAMIENTOS PARA SU ABORDAJE
}

\author{
SUICIDE AT THE COMMUNITY LEVEL: GUIDELINES FOR ITS \\ APPROACH
}

\author{
Adriana Maroto Vargas $^{1}$
}

Fecha de recepción: 24 de abril de 2017 - Fecha de aceptación: 19 de junio de 2017

\begin{abstract}
Resumen
El suicidio, desde una aproximación psicosocial, debe llamar la atención sobre las dinámicas sociales, las interacciones interpersonales, las redes vinculares y los aspectos estructurales asociados. Por lo tanto, se deben pensar estrategias comunitarias para su abordaje. Como resultado de un proyecto de investigación, que hizo revisión bibliográfica y consultó a profesionales con experiencia en el abordaje del suicidio, se presentan algunos lineamientos para orientar el trabajo en comunidad. Se proponen la investigación centrada localmente, el respeto a la diversidad cultural, el rol de los servicios de salud en el primer nivel de atención y algunas características básicas de un programa. Sin embargo, como principales hallazgos se enfatiza la importancia de articular modelos interdisciplinarios y multisectoriales de trabajo, lo cual implica una revisión de la vinculación que ha prevalecido entre las instituciones y las comunidades, a fin de garantizar a las personas y los grupos organizados una participación activa en la identificación de su problemática y la forma de resolverla. En segundo lugar, las iniciativas deben sostenerse en pilares de puertas abiertas, abordajes grupales y sin la interferencia de requisitos administrativos. Finalmente, se destaca la escucha activa y el fortalecimiento del vínculo. La escucha puede ser ofrecida tanto profesionalmente como por otras personas de la comunidad. El reto es identificar qué espacios de escucha ya existen, ampliar, diversificar e innovarlos. Lo anterior está intrínsecamente relacionado con el vínculo, ya que un lazo social válido permite una red de soporte frente al riesgo suicida. Palabras clave: Suicidio, Comunidad, Enfoque interdisciplinario, Vínculo, Escucha activa.
\end{abstract}

\begin{abstract}
Suicide, from a psychosocial approach, should draw attention to social dynamics, interpersonal interactions, linking networks and associated structural aspects. Therefore, community strategies should be considered for their approach. Some guidelines are presented to orient the work in community as a result of a research project that did bibliographic review and consulted professionals with experience in the approach to suicide. The propose includes locally focused research, respect for cultural diversity, the role of health services in the first level of care and some basic characteristics of a program. However, the main findings emphasizes the importance of articulating interdisciplinary and multisectoral models, which implies a revision of the linkage that has prevailed between institutions and communities in order to guarantee, to individuals and organized groups, an active participation in the identification of its problems and the way to solve them. Second, initiatives must be supported by open-door pillars, group approaches and without the interference of administrative requirements. Finally, active listening and strengthening of the link are highlighted. Listening can be offered by professionals and by other people
\end{abstract}

1 Escuela de Psicología, Facultad de Ciencias Sociales, Universidad de Costa Rica, adriana.maroto_v@ucr.ac.cr 
in the community. The challenge is to identify what listening spaces already exist, expand, diversify and innovate them. This is intrinsically related to the social link which allows a support network against suicidal risk.

Key Words: Suicide, Community, Interdisciplinary approach, Link, Active Listening.

\section{Introducción}

Este artículo presenta los resultados del tercer objetivo específico del proyecto de investigación Debates actuales en torno al suicidio: alcances teóricos y propuestas de abordaje realizado durante el año 2016. El objetivo general de la investigación fue contribuir a la discusión en torno al entendimiento teórico del suicidio, sus estrategias de prevención y abordaje en el país. En este texto se presentan estrategias de abordaje del suicido desde un enfoque comunitario, con lo cual se espera realimentar iniciativas que aborden el tema, incluyendo proyectos de acción social de la Universidad de Costa Rica.

La investigación tuvo un abordaje metodológico cualitativo, el cual se caracteriza por construirse en el proceso mismo de la investigación y por lo tanto, por su flexibilidad para hacer las adaptaciones necesarias en función de los cambios que experimente el objeto de estudio (Rodríguez Gómez, Gil Flores y García Jiménez, 1996). Asimismo, tuvo un diseño exploratorio, cuyo fin es aportar a la comprensión teórica y las propuestas de abordaje del suicidio desde abordajes que trasciendan el modelo clínico de enfoque medicocéntrico, individual e institucional, el cual ha predominado en el país según los hallazgos del proyecto realizado en el 2014 (Maroto, 2015).

El estudio realizó un exhaustivo trabajo de revisión y análisis de ocho documentos vinculados a códigos de ética profesional y política pública, 60 publicaciones (principalmente artículos científicos), cinco entrevistas a profesionales (cuatro individuales y una grupal) y un grupo de discusión (10 profesionales). Tanto las entrevistas como el grupo de discusión, previo consentimiento informado de las personas consultadas, se grabaron y transcribieron para su análisis, el cual fue realizado mediante la técnica de análisis crítico de discurso. Desde esta técnica interesa realizar una indagación analítica sobre el discurso respecto al poder que se ejerce por parte de grupos e instituciones. Este genera una desigualdad, la cual es practicada, reproducida o combatida por el texto. Por ende, el análisis debe tener un referente social y político, además de su aspiración de tomar una posición de resistencia. (Van Dijk, 1999)

En primer lugar el artículo presenta algunas apreciaciones generales sobre el abordaje conceptual del suicido desde un enfoque psicosocial, el cual permite una coherencia epistemológica con los lineamientos para el abordaje comunitario, principal objetivo de este texto.

\section{El suicidio desde una perspectiva psicosocial: la importancia del vínculo}

El suicidio ha sido catalogado como un problema de salud pública debido a su tendencia creciente a nivel internacional. Esto, más que centrar la atención en las personas de manera individual debe llamar la atención sobre las dinámicas sociales, incluyendo no solo las interacciones y redes vinculares sino también los aspectos estructurales vinculados al mismo. (Corpas, 2011; Martínez, 2008; Pérez, 2014; UNICEF, 2012)

Desde finales del siglo XVIII Durkheim ubicó el suicido como un hecho social y estableció bases teóricas para entender y estudiar el tema en el marco de las dinámicas sociales. No obstante, han prevalecido los enfoques clínicos en el abordaje de la temática a pesar que desde la psicología no es posible pensar al sujeto al margen del otro y su contexto, por lo tanto el sujeto inexorablemente es parte de una red vincular, de una sociedad y de una cultura. Profesionales consultados para este trabajo coinciden en que el suicidio debe ser estudiado en el marco de las redes vinculares. (Ana Jurado; Lucía Molina, José Manuel Salas y Kira Schroeder, comunicación personal, 2, noviembre, 2016) "Necesariamente 
tiene que ser vincular, a pesar de que en algún caso alguien pueda decir que es una cuestión como muy de un sujeto intrapsíquicamente. Hay una red vincular necesariamente asociada." (José Manuel, Salas, comunicación personal, 2, noviembre, 2016).

Ivannia Chinchilla (comunicación personal, 2, noviembre, 2016) refiere que el sufrimiento se construye desde lo relacional a la luz de otros que han estado presentes y que han dotado esos vínculos de significados desde los primeros años de vida. Aun cuando la persona no esté en posibilidad de verbalizarlo, es en ese proceso de socialización que se ha construido lo que es o no importante, incluyendo la identificación del dolor y el sufrimiento, entre muchos otros aspectos.

Por lo tanto, la ruptura de esos vínculos incide en el suicidio. Según Gonzales, Gil, Hernández y Henao (2016) este es una opción cuando la persona ha distorsionado la responsabilidad de demandar la reestructuración de los vínculos rotos con otras personas que le son significativas. Y esto es posible gracias a crisis, tanto a nivel global como local que impacta en las formas de organización familiar y la capacidad de respuesta frente a estas situaciones.

La familia y el grupo de pares suelen ser los primeros referentes al pensar en redes de apoyo, sin embargo estos grupos pueden cumplir un papel tanto como factor protector como factor de riesgo, depende de si brindan contención y apoyo o más bien son violentos y generan expulsión. (Lucía, Molina, comunicación personal, 2, noviembre, 2016) Entonces es necesario ampliar la perspectiva ya que la red vincular no solo hace referencia a los contactos personales y familiares, tales como grupos de pares, familia cercana, compañeros y compañeras de trabajo, entre otros, sino también a las dinámicas comunitarias y sociales en general. Para Elizabeth Seaward y Ana Jurado (comunicación personal, 2, noviembre, 2016) ese vínculo social refiere a múltiples aspectos, tales como los patrones sociales aprendidos, el sentido de pertenencia frente al mundo, el vínculo con el lenguaje, las tradiciones del grupo de referencia, entre otros. Esta no intenta ser una lista indicativa, sino enfatizar el entendimiento del vínculo en un sentido muy amplio, ya que este brinda sentido de pertenencia e identidad.

Consiguientemente, entender el suicidio desde esta aproximación teórica implica pensar estrategias de abordaje que trasciendan el espacio de atención clínica individual. En este artículo interesa plantear una propuesta de algunos lineamientos para el trabajo desde una aproximación comunitaria.

\section{Lineamientos para el abordaje comunitario del suicidio}

Hablar de lo comunitario requiere precisiones conceptuales que trascienden el objetivo de este artículo; el concepto de comunidad no se agota en una delimitación geográfica sino que esencialmente refiere a un espacio vincular. La comunidad es un proceso colectivo construido, gestado y vivido de una forma dinámica, no debe ser entendida necesariamente como un colectivo homogéneo, además de la pertenencia y los vínculos, también puede existir el conflicto, el disenso y la exclusión. (Dobles, En prensa; Escalante, En prensa)

Las palabras tienen esta posibilidad que nos permiten hablar pero también remiten a otra cosa, ¿qué es lo comunitario? Yo tal vez empezaría por decir, pensemos en lazos válidos, cuando les decía a ustedes que los muchachos a veces no encuentran en la familia ni en ciertos grupos comunitarios, apoyos o redes como solemos decir, también yo tendría que decir ¿cuáles son los lazos válidos? (Lucía, Molina, comunicación personal, 2, noviembre, 2016).

El abordaje comunitario tiene gran relevancia porque permite fortalecer las estrategias de promoción de la salud y la prevención del suicidio, con lo cual se pueden ver resultados a mediano y largo plazo. Laura Chacón (comunicación personal, 29, septiembre, 2016) afirma que la institucionalidad históricamente se ha preocupado por las cifras de expulsión social evidenciadas en diferentes indicadores sociales; sin embargo, sigue enfocando los esfuerzos en el abordaje individual como respuesta a esas situaciones críticas. 
Hemos llegado al agotamiento de la atención casuística, porque la atención casuística puede servir y yo sigo teniéndola si tengo otros externos que ayudan, pero hemos llegado a cada condición de olvido a múltiples comunidades y a tal condición de no poder saber cómo se maneja la infancia y adolescencia, que si no somos un colectivo grande de red, de sostén, vamos a llegar a condiciones cada vez más complicadas (Laura, Chacón, comunicación personal, 29, septiembre, 2016).

Si el suicidio no necesariamente obedece a un deseo de muerte, sino a una resolución de un dolor emocional insoportable para la persona, es muy pertinente la siguiente pregunta planteada por Rocío Murillo ¿cuál es la responsabilidad de lo social en brindar opciones que le permitan a una persona ubicar una salida o una alternativa al suicido en un momento de crisis? (Comunicación personal, 2, noviembre, 2016) Debe notarse que el énfasis se pone en lo colectivo, no en la persona en riesgo ni su entorno más inmediato.

Según Jaime Carmona (comunicación personal, 9, septiembre, 2016) trabajar en la prevención del suicidio implica enfocar los esfuerzos en la calidad y la calidez de los vínculos. Esto refiere a promover dinámicas incluyentes, fortalecer las redes vinculares, el examen crítico y la intervención oportuna de ciertas tensiones vinculares inevitables y necesarias, así como brindar participación a cada persona en los asuntos que le implican fundamentalmente.

Existen pocos registros de experiencias de abordaje comunitario del suicidio, tanto a nivel nacional como internacional. Este artículo aporta algunos principios generales para orientar y articular este tipo de iniciativas, las cuales deben tener como objetivos, por un lado la promoción de la salud y la prevención del suicidio pero por otro lado la detección del riesgo y la articulación de una respuesta oportuna frente a la conducta suicida. "Lo más importante en estrategias comunitarias es fortalecer las capacidades de detección comunitaria de una intencionalidad suicida en ese sentido, o sea que las comunidades puedan abiertamente hablar del tema." (Ana, Jurado, comunicación personal, 2, noviembre, 2016).

\section{Promoción de la salud y prevención del suicidio}

Acorde con la Organización Mundial de la Salud (2016), la promoción de la salud mental se orienta por acciones tendientes a crear entornos y condiciones de vida que permitan modos de vida saludables como una estrategia sostenible en el tiempo y que busca resultados a largo plazo. Según Rocío Murillo (comunicación personal, 2, noviembre, 2016) en nuestro país no existe una cultura de promoción de la salud mental y al contrario, persisten mitos y estigmas de gran peso referentes a la consulta psicológica, la cual se entiende como un signo de locura y no como la búsqueda de un espacio de apoyo y contención. Ubicándose en el ámbito comunitario, José Manuel Salas (comunicación personal, 2, noviembre, 2016) identifica la habilitación de espacios de escucha como una estrategia de promoción de la salud, eje que se abordará posteriormente. Dicho en palabras de Mauricio Campos (comunicación personal, 25, agosto, 2016), la aspiración desde la salud mental comunitaria "sería trabajar no solamente con chicos enfermos sino que trabajáramos para que no se enfermaran."

Centrando la atención en el eje de prevención, especialmente con adolescentes, una estrategia identificada es la promoción y el fortalecimiento de habilidades para la vida. Como parte de ellas, Ivannia Chinchilla (comunicación personal, 2, noviembre, 2016) incluye la consolidación de estrategias de manejo de emociones y la resolución de problemas de la vida, entre otros.

Otra estrategia es el trabajo en la deconstrucción de mitos y tabúes, de tal manera que sea un tema del cual se pueda hablar abiertamente y sea posible identificar personas en riesgo suicida sin menospreciar la situación reportada (Mauricio, Campos, comunicación personal, 25, agosto, 2016; Lilliana, Mejías, comunicación personal, 2, noviembre, 2016). Asimismo, la capacitación a diferentes actores institucionales y comunitarios en primeros auxilios psicológicos permitiría no solo un primer nivel de acompañamiento a la persona, sino también una referencia adecuada. 
(...) si logramos que en las escuelas en los colegios en las instituciones hayan personas preparadas para escuchar de una manera abierta, de una manera serena, de una manera crítica, de una manera madura a personas en riesgo suicida, sin juzgarlas, sin sermonearla, sin retarlas, sin hacer apología, sino de una manera serena y crítica. Yo estoy seguro que eso puede significar los primeros auxilios psicológicos que permitan hacer una derivación para que la persona reciba una atención especializada. (Jaime, Carmona, comunicación personal, 9, septiembre, 2016)

\section{Investigación centrada localmente}

Coincidente con lo encontrado en un estudio previo, uno de los primeros requisitos para desarrollar una estrategia comunitaria es realizar algún tipo de diagnóstico o investigación para conocer dinámicas locales vinculadas con el suicidio, los recursos disponibles para trabajar y las estrategias utilizadas previamente. Además, este trabajo permite visibilizar y posicionar el tema a nivel local, el cual suele estar invisibilizado en el espacio público a pesar de su presencia en la vida cotidiana. (Maroto, 2015)

La investigación o el diagnóstico deben orientarse de acuerdo a los recursos y los intereses de las personas o instituciones a cargo del seguimiento a la iniciativa. No obstante, es importante indagar sobre los factores de riesgo y los factores protectores específicos a cada comunidad, ya que estos deben orientar el trabajo.

\footnotetext{
Una variable que se constituya como factor de riesgo en un lugar, no quiere decir que ese resultado sea aplicable a otro colectivo sin medición previa. De la misma manera que lo que hoy es válido para un determinado contexto cultural no necesariamente lo consolida como factor fijo en el paso de los años, ya que estamos hablando de productos cognitivos individuales y colectivos que están en permanente movilidad, dada su alta relatividad axiológica (Martínez, 2008, p. 3).
}

Entre los factores protectores es vital identificar la red social que puede brindar soporte a la persona. Según Mauricio Campos (comunicación personal, 25, agosto, 2016) a fin de prevenir el riesgo suicida a futuro se debe indagar en las estructuras vinculares en que la persona se siente apoyada, de esa manera es mucho más probable identificar las herramientas para no encontrarse en la misma situación. No obstante, Lilliana Mejías (comunicación personal, 2, noviembre, 2016) advierte que esta valoración se debe realizar en cada caso y no se puede partir de principios generales tales como "la familia es un factor protector".

A veces la familia no constituye una red, más bien si fuera por la familia esa persona estaría muerta ya, a veces el grupo social o de trabajo tampoco, entonces a veces esas que solemos estereotipar como redes, están llenas de huecos, es decir es como que el trapecista se tire en la red y se cayó (Lilliana Mejías, comunicación personal, 2, noviembre, 2016).

Los factores protectores son fundamentales para trabajar la promoción y prevención de la salud mental, sin embargo se ha estudiado poco al respecto. Desde el referente teórico sustentado en esta investigación las redes vinculares (pares, familia, comunidad, entre otros) cumplen un rol fundamental en esta línea. A partir de su experiencia de trabajo en Pavas, Laura Chacón afirma “estoy convencida de la comunidad como posibilidad de sostén" (Comunicación personal, 29, septiembre, 2016).

Un antecedente de este tipo en el país es el diagnóstico realizado en el cantón de Dota en el 2014, este fue la base para formular la propuesta de trabajo en esa comunidad. En este proceso, los resultados apuntaron a identificar dinámicas comunitarias vinculadas a la moral y los valores (control social, los mandatos tradicionales de género, la violencia sexual y las problemáticas familiares), así como la ubicación geográfica del cantón y las pocas actividades en qué invertir el tiempo libre como factores de riesgo. Por su parte, las redes de apoyo se identificaron como un factor protector de gran relevancia. (Castillo y Maroto, 2014) 
En los factores de riesgo presentados por Martínez (2008) a partir de la experiencia de trabajo en la región de la Patagonia en Argentina, se da una especial importancia a la ruptura de vínculos, sean familiares, de amistad, afectivas o escolares; así como la marginación social. Esto es coincidente con el análisis teórico presentado anteriormente.

\section{Participación multisectorial e interdisciplinaria}

El suicidio, como fenómeno complejo, debe ser abordado de manera integral y sistemática por parte de la sociedad, lo cual implica un tratamiento multisectorial e interdisciplinario. La experiencia ha mostrado que este es el único medio por el cual se pueden alcanzar avances sustanciales y sostenibles orientados a la prevención del suicidio. (Guibert, 2002)

Jaime Carmona (comunicación personal, 9, septiembre, 2016) recuerda que la base de la interdisciplinariedad es la disciplinariedad, por lo cual cada profesional debe ubicarse en su área y aportar desde su conocimiento, su rol y su discurso, pero a la vez preguntarse “¿de qué manera puedo dialogar con el comunicador, con el educador, con el trabajador social, con el médico? y ¿de qué manera puedo incluir los aporte de esas disciplinas a mi intervención?, y por supuesto también construir estrategias colectivas."

Por lo tanto, tal y como lo enfatiza Carmona, implica reconocer que desde las diferentes disciplinas se tiene un conocimiento el cual coadyuva a abordar el tema y por ende profesionales de diferentes áreas tienen alguna autoridad para intervenir. Esto establece, la voluntad de construir lazos como una condición básica de trabajo, tarea posible solo si se rompe con los lenguajes especializados y se buscan formas de interlocución. Mauricio Campos (comunicación personal, 25, agosto, 2016) asevera desde su experiencia de trabajo "lo bonito de hacerlo interdisciplinario es que todos lo vemos desde una perspectiva diferente, y hemos ido entendiendo que en el conjunto de todos, sentimos mejor los casos."

La participación multisectorial refiriere al involucramiento de actores comunitarios muy diversos. Se presentan los que fueron identificados en el proceso de consulta:

- Municipalidades. Tienen un rol fundamental en la articulación de actores a nivel local y la implementación de estrategias orientadas a la promoción de la salud y el fortalecimiento de la identidad y los vínculos comunitarios.

- Personal de salud. El personal del primer nivel de atención suele conocer a la población y además cumplen un rol fundamental en la articulación con los otros niveles de atención en salud.

- Personal especializado en salud mental, e idealmente capacitado en el manejo de la conducta suicida. Incluye tanto el personal vinculado a las instituciones como aquellas personas que son de la comunidad y pueden realizar aportes importantes, tanto en su trabajo profesional (por ejemplo atención clínica, articulación de grupos de apoyo) como trabajo voluntario.

- Instituciones educativas. Estas han sido identificadas como un lugar preferencial para el abordaje del suicidio en tanto las personas menores de edad son una población considerada prioritaria por tener mayor riesgo suicida. Además, es un espacio clave para el desarrollo psicosocial: tiene incidencia tanto sobre competencias sociales, el plano cognitivo, la configuración de la identidad, el grupo de pares y la red de apoyo, entre otros. Asimismo, desde estas se facilita la referencia hacia los centros de salud en caso de ser necesario. (Chávez, Medina y Macías, 2008; González, Arana y Jiménez, 2008; Mosquera, 2016; Piedrahita, Paz y Romero, 2012; Valadez, Amezcua, González, Montes y Vargas, 2011).

- Personal de primera respuesta (cruzrojistas y policías). Esta población se enfrenta directamente el tema en la atención de las emergencias pero usualmente no tiene la capacitación requerida en esta temática específica. 
- Organizaciones no gubernamentales que trabajen directamente el tema. Son actores importantes porque suelen tener personal capacitado y experiencia en el desarrollo de diversas estrategias. No obstante, pocas veces tienen presencia a nivel local por limitaciones económicas.

- Iglesias (tanto sus líderes religiosos como grupos organizados). Pueden brindar un aporte como red de apoyo para personas en riesgo y familias en momentos de crisis.

- Cualquier grupo organizado de la comunidad. Independientemente del eje que reúna a un grupo, este se puede convertir en red de apoyo para una persona con riesgo suicida. Pueden ser grupos relacionados a actividades culturales y artísticas, deportivas, empoderamiento de mujeres, ambientales, entre muchos otros.

- Población en general. Son actores fundamentales. Es preciso mantener estrategias que fomenten la participación activa de personas interesadas y comprometidas. De esta forma se amplían las posibilidades de identificar y referir de manera oportuna las personas con riesgo suicida.

La participación de esta diversidad de actores a nivel local es un eje central y debe ser entendida como el involucramiento de todos ellos en la toma de decisiones, el control y la responsabilidad con respecto a la atención de la salud, no como una participación en actividades ya organizadas o como usuarios de servicios. (Guibert, 2002) Esto otorga a todas las instituciones un papel importante en el fomento de las diferentes estrategias de participación social, más allá del servicio brindado en la comunidad.

En este sentido se debe revisar muy críticamente el rol de las instituciones y sus profesionales, asumiendo una posición de encuentro de saberes tanto con las personas de la comunidad como con profesionales de otras áreas.

\footnotetext{
Yo creo que desidealizar la condición de profesionales, dejar de creer que si nosotros no hacemos algo nadie lo puede hacer, dejar los egoísmos disciplinares, de que, en el sentido pues de abrirnos a intercambiar y a interactuar con las demás profesiones y a aportar desde nuestra profesión para que los otros también puedan hacer desde las propias. Como lo diría, democratizar un poco el conocimiento, para que esas cosas que nosotros sabemos, pues hay algunas realmente demasiado especializadas que quizás pues no son asequibles a mucha gente, pero hay muchas cosas que nosotros sí podemos ayudar a que lleguen y a traducir y a hacer un gran esfuerzo didáctico en este campo específico. (Jaime, Carmona, comunicación personal, 9, septiembre, 2016).
}

Si se parte de un proceso participativo, no debe existir un modelo definido previamente, sino coordenadas orientadoras del trabajo, pero es a partir de ese encuentro con los diferentes actores comunitarios y en un trabajo creativo, que se van formulando y construyendo las propuestas. (Allan, Rímola, comunicación personal, 11, octubre, 2016; Elizabeth, Seaward, comunicación personal, 2, noviembre, 2016) Deben reconocerse y validarse las formas de organización existentes y valoradas en términos positivos por la misma comunidad.

Muchas veces no es lo oficial lo que está funcionando, o sea, no es el presidente de la junta directiva de la asociación o de la cooperativ que tiene el puesto o las instituciones oficiales las que están funcionando (Kira, Schroeder, comunicación personal, 2, noviembre, 2016).

\section{Respeto a la diversidad cultural}

En relación estrecha con el eje anterior, un modelo de abordaje comunitario de la promoción de la salud en general o de prevención del suicidio en particular, debe respetar las pautas culturales tanto en la construcción de las propias categorías de salud y enfermedad existentes en la comunidad, así como las estrategias de organización, participación, acompañamiento y atención que ya han sido exitosas y responden a patrones culturales propios. Esto tiene una significación muy particular cuando se trabaja en comunidades indígenas, sin embargo debe ser un principio general. 
Si se parte de ese respecto a la diversidad cultural de la comunidad con que se está trabajando, se facilita la apropiación del proceso por parte de actores locales, quienes formulan sus propias soluciones al problema identificado, las cuales son más sostenibles. "Cada pueblo y cada cultura debe tener formas propias de afrontar el suicidio y éstas deberían ser incorporadas a los protocolos de las instituciones a cargo de la salud" (UNICEF, 2012, p. 205).

\section{El rol de los servicios de salud del primer nivel de atención}

El suicidio se ha tendido a abordar en hospitales especializados y centralizados en áreas urbanas, en el caso de Costa Rica los casos suelen derivarse a los hospitales psiquiátricos ubicados en San José. Por lo tanto, la labor del primer nivel de atención se ha visto prácticamente reducida a la referencia de casos, sin ni siquiera tener un seguimiento de estos cuando las personas regresan a la comunidad o de las personas sobrevivientes ${ }^{2}$, según corresponda.

No obstante, la intervención de trabajadoras y trabajadores del primer nivel de salud es muy estratégica porque generalmente tienen una vinculación mucho más directa con la comunidad.

Son articuladores de ésta con el sistema médico asistencial, en muchos casos se constituyen en la fuente de los primeros cuidados sanitarios para la población y, al estar insertos en las pautas culturales del lugar, son conocedores de las redes de sostén y de apoyo psicológico validadas por la población, para paliar la crisis (Martínez, 2008, p. 6).

Consecuentemente, se convierten en actores fundamentales para trabajar en estrategias que trascienden el modelo de atención clínica e individual y propicien una mayor cercanía con otros actores comunitarios y de esta manera lograr mejores resultados en el mediano y largo plazo.

\section{Algunas características básicas de los proyectos o programas}

Resultado de la experiencia de trabajo de la investigadora en el tema se han identificado dos proyectos con enfoque comunitario que brindan importantes lecciones aprendidas. Uno de ellos es conocido como "Casas de Escucha" en Pavas ${ }^{3}$, proyecto coordinado por la Dra. Laura Chacón Echeverría, y el otro el Centro de Intervención en Crisis y Rehabilitación Psicosocial ${ }^{4}$ en la Patagonia Argentina, coordinado por el Lic. Carlos Martínez. Este último es el único que tiene el suicidio como eje específico de trabajo. Estas dos experiencias tienen algunas características que son identificadas como prioritarias tanto por Laura Chacón (comunicación personal, 29, septiembre, 2016) como por Martínez (2008), las cuales se presentan a continuación como recomendaciones para la implementación de proyectos.

- Un espacio físico propio. Este espacio debe ser independiente de otros servicios, ser un espacio "acogedor" y de muy fácil acceso a la población meta. Para esto se deben evitar barreras económicas (gastos por traslado) y sociales (el estigma de ser parte de un servicio de salud mental en un hospital por ejemplo). Esto brinda un referente a las personas de dónde pueden acudir en caso de requerir ayuda, así como una apropiación como una iniciativa local.

- Tener una política de "puertas abiertas". Si bien se atiende población con un perfil muy definido e incluso se puede realizar algún proceso de tamizaje o evaluación del riesgo, las personas pueden

2 Se identifica como sobreviviente de suicidio a una persona que tenía un vínculo cercano con quien murió por esta causa.

3 Son espacios gestionados en una alianza público-privada en que participan la Fundación Mente Sana (conocida como Fundamentes), el Hospital Nacional Psiquiátrico, el Ministerio de Educación Pública, la Municipalidad de San José y las asociaciones comunales de Pavas. Las casas son centros de atención diurna dirigidos a menores de edad en situaciones de alto riesgo psicosocial. El objetivo es procurar su permanencia dentro del sistema educativo costarricense.

4 Es un centro especializado en atención de riesgo suicida en el sistema de salud público de Argentina. Está adscrito al Hospital Regional de Río Gallegos, ubicado en la provincia de Santa Cruz, Argentina. 
acudir a solicitar un espacio sin necesidad de tener una cita previa o realizar algún trámite formal. Una primera atención, aunque sea informativa, se brinda de manera inmediata y se establecen parámetros para el seguimiento o la referencia si es del caso.

- No se solicitan requisitos institucionales para el ingreso. Ambos servicios tienen vinculación al sistema público de salud, sin embargo, no requieren que las personas cumplan requisitos formales como tener un seguro social, por lo tanto, si una persona cumple con el perfil establecido, se atiende dentro del modelo sin pedirle otros requisitos administrativos.

- Atención interdisciplinaria. El modelo tiene una base interdisciplinaria de acuerdo a los objetivos establecidos (prevención o atención). Sin embargo, ninguno de los dos tiene la atención centrada en el modelo médico ni farmacológico, el énfasis está en la escucha, el acompañamiento y el fortalecimiento del vínculo. El acompañamiento se realiza tanto presencial durante la visita de la persona al centro, como telefónico o en visitas a la casa en caso de ser requerido.

Atención grupal. El énfasis es gestionar espacios grupales, tanto de actividades de promoción de la salud como terapéuticos.

\section{La escucha en el ámbito comunitario}

En el proceso de consulta se encontraron dos elementos, intrínsecamente relacionados, fundamentales en el abordaje del suicidio, sea a nivel clínico o a nivel comunitario: la escucha y el vínculo. Estos se convierten en los elementos mínimos de un proyecto comunitario para la atención del suicidio.

Desde su experiencia en la comunidad de Pavas, para Laura Chacón (comunicación personal, 29, septiembre, 2016), "lo importante es escuchar, entonces por eso se llaman Casas de escucha, porque lo importante es escuchar eso que traemos adentro y que nos destruye de tal forma que hace tener la muerte como opción".

La pregunta es si se trata de una escucha de una o un profesional en salud mental o una escucha de un otro, independientemente de quien sea. Al respecto hay diferentes criterios. En este artículo, más que divergentes, se entienden como las alternativas de espacios de escucha a habilitar. Para José Manuel Salas (comunicación personal, 2, noviembre, 2016) es necesario tener dispositivos de escucha al alcance de todas las personas, estos pueden ser las familias, las instituciones o las mismas comunidades. No obstante, para tener el potencial de propiciar vínculos que tengan una incidencia importante en una persona con ideación suicida deben ser estructuras amorosas de escucha.

Por lo tanto, afirma Kira Schroeder (comunicación personal, 2, noviembre, 2016) en el ámbito comunitario se debe indagar ¿quién o quiénes están cumpliendo este rol de escucha? Estos serían actores fundamentales a involucrar en las estrategias para la prevención del suicidio.

Lo anterior, no desestima el lugar de la escucha ofrecido por profesionales en salud mental; según Schroeder, si estos espacios de escucha estuvieran habilitados a nivel familiar o comunitario no existirían altas tasas de suicidio en algunas localidades en particular. Esto interroga de manera directa el rol de las y los profesionales en psicología en estas comunidades, quienes deberían habilitar espacios para canalizar estas demandas.

\footnotetext{
Ahí es donde tenemos que hacer valer lo que nosotros hacemos, es una escucha diferente, la tendría que hacer un psicólogo, que no es la misma escucha que están dando en la iglesia, o en los grupos, o en el colegio, es decir, esta es una escucha diferente, y yo pienso que muchas de las intervenciones que se hacen se orientan un poco a tapar la boca y "vea que usted tiene esto y tiene lo otro" y yo creo que lo que hay que hacer es abrirle la boca, lo que hay que provocar es que tenga por lo menos ganas de ir a hablar (Kira, Schroeder, comunicación personal, 2, noviembre, 2016).
}

Según Laura Chacón y Dagoberto Solano (comunicación personal, 29, septiembre, 2016 y 2, noviembre, 2016 respectivamente) la escucha profesional es un gran reto debido a la limitada experiencia de la población en estos espacios. Las personas menores de edad tienden a evitar el espacio psicológico porque lo confunden con la experiencia del regaño que usualmente reciben de las personas adultas, 
incluyendo tanto sus familias como las instituciones educativas. Sin embargo, a partir de la experiencia se logra cambiar el imaginario. Solano refiere comentarios recibidos por parte de sus pacientes en un hospital público tales como es la primera vez que alguien "me escucha", "no me juzga", "me entiende." Si bien es un proceso lento, a partir de estas experiencias se puede aprender a demandar este espacio y hasta se podrían fomentar nuevas dinámicas en espacios personales y familiares.

En el ámbito comunitario estas posibilidades de escucha, tanto las brindadas por profesionales como por otros actores, se deben ampliar, diversificar e innovar. Según Laura Chacón (comunicación personal, 29, septiembre, 2016) el arte es un medio esencial para propiciar el reflejo del yo y por tanto, la posibilidad de suturar la ruptura a través de un eje creativo.

\section{El fortalecimiento del vínculo en el ámbito comunitario}

La escucha es un eje fundamental, pero además debe brindarse una opción de sostén a la persona en riesgo suicida. El vínculo es un tema complejo, partir de su análisis teórico trasciende los objetivos de este artículo, sin embargo sí es importante plantear algunas reflexiones sobre el fortalecimiento del vínculo en los procesos de prevención del suicidio.

Según Jaime Carmona (comunicación personal, 9, septiembre, 2016) los comportamientos autodestructivos no se pueden entender o explicar solamente desde la dinámica interna del psiquismo de un ser humano. La subjetividad se nutre y se constituye a partir de las interiorizaciones que hacemos de los vínculos con los otros, de tal forma que en la subjetividad de todo ser humano está interiorizada la estructura social. Por lo tanto, afirma este psicólogo colombiano

Tenemos que aprender, en el campo de la investigación y la teorización de los comportamientos autodestructivos, a tender ese puente que va desde lo subjetivo de un ser humano que está atormentado a punto de quitarse la vida y su estructura social y su contexto familiar y su contexto de interacciones, sus vínculos con su pareja, con su familia, con sus partenaires digamos en las interacciones sociales, etcétera.

Desde su experiencia con grupos operativos se producen efectos de alivio y de bienestar subjetivo de las personas que participan, incluyendo quienes tienen pensamientos autodestructivos. Afirma, "si uno logra tocar ese contexto vincular fundamental de una persona en riesgo suicida, pasan cosas realmente sorprendentes" (Jaime, Carmona, comunicación personal, 9, septiembre, 2016).

¿De qué vínculo se habla? ¿Es un vínculo con un otro significativo? ¿Es un vínculo familiar? ¿Es un vínculo con un entorno más amplio? En algunos casos, se puede tratar de otros significativos presentes en la vida de la persona desde su infancia, y a través de lo relacional han dotado muchos significados de desvitalización (Ivannia, Chinchilla, comunicación personal, 2, noviembre, 2016). Los vínculos son tanto un factor protector o como un factor de riesgo, es así como las intervenciones psicosociales deben orientarse a fortalecerlos.

Tener un otro de sostén permite una posibilidad de reciprocidad y en ese sentido, la comunidad es fundamental para el crecimiento del ser humano, y este es uno de los principios de las Casas de Escucha coordinadas por Chacón en Pavas. La vida en comunidad propicia que "ese ser humano va a crecer y va a tener una razón para vivir (...) porque si se da se devuelve, el ser humano es hermoso en ese sentido, pero si no se da no hay nada que devolver" (Laura, Chacón, comunicación personal, 29, septiembre, 2016). Por ende, el suicidio consumado refiere a una ausencia de ese soporte.

Por lo tanto, como se ha planteado anteriormente, debe pensarse en lazos válidos y no en lugares comunes como la familia, porque justamente estos grupos sociales más cercanos pueden no entender las circunstancias de la persona y más bien contribuir a generar la crisis suicida. (Lucía, Molina, comunicación personal, 2, noviembre, 2016)

Laura Chacón (comunicación personal, 29, septiembre, 2016) ofrece un ejemplo concreto de cómo trabajar en el fortalecimiento del vínculo y de una red de soporte para la persona. 


\begin{abstract}
Por ejemplo ahora tengo una chica con muchas cartas suicidas, entonces yo voy al colegio, trabajo con la orientadora o el psicólogo de ese colegio (...) esta chica, ya hablé con el profesor de teatro. Nunca dejo lo que es la familia por fuera, a veces la familia, si no sirve papá o no sirve la mamá por distintas situaciones, hablo con una tía, una abuela, realmente vuelvo a invitar a que ese entorno intente cambiarse. Por el otro lado, (...) en Pavas tengo mejores instrumentos de lo que tengo aquí en la consulta clínica. Porque (...) somos varias psicólogas que podemos escuchar algún día algún dolor o alguna tentativa suicida o alguna ideación etc. Pero inmediatamente nosotros ya en nuestras casas tenemos los programas de telas, de danza, de cocina, de arte, de teatro, no tengo que buscarlo. (...)Tenemos un grupo de profesores con mucha fuerza entonces inmediatamente damos una fuerza de sostén (...). Simplemente sabemos que hay una vulnerabilidad entonces es ahí con un ejército que entramos.
\end{abstract}

El trabajo en comunidad implica un compromiso ético, una lectura de los procesos, propuestas creativas y el involucramiento de muy diversos actores sociales. Estas propuestas se deben entender como lineamientos orientadores, sabiendo que cada experiencia puede llevar a procesos y resultados muy diversos.

\title{
Conclusión
}

El abordaje comunitario del suicidio se considera una estrategia prioritaria para abordar esta temática, esto no implica desconocer la importancia del trabajo clínico e individual, sino reconocer sus limitaciones. Por un lado, el restringido acceso a las consultas psicológicas, tanto por su costo a nivel privado, el modelo de la Caja Costarricense del Seguro Social que condiciona la solicitud de la consulta a una referencia médica y el reducido número de profesionales en psicología e incluso psiquiatría por cada área de salud, así como el estigma social de las implicaciones de acudir a consulta psicológica. Y por otro, si a nivel teórico se entiende el suicidio como parte de una estructura vincular, incluyendo desde los grupos de referencia más cercanos hasta las estructuras sociales, es preciso trascender el abordaje del tema hacia un enfoque comunitario. A su vez, este permite, pensar en estrategias de promoción de la salud, que además de anticipar las crisis y generar espacios de contención y acompañamiento en la vida cotidiana de las personas, trabaja sobre estrategias orientadas a fortalecer los entornos y las condiciones para una vida saludable como una estrategia sostenible en el tiempo.

El abordaje comunitario implica grandes retos desde su diseño hasta su implementación. En algunas ocasiones tiende a pensarse lo comunitario como un traslado de los servicios de salud mental al primer nivel de atención. Si bien esto sería un avance importante desde la salud pública, es solo uno de los elementos a articular desde este enfoque, caso contrario estaría persistiendo el abordaje casuístico e institucionalizado. El artículo presentó algunos lineamientos a seguir tales como partir del conocimiento de cada localidad, lograr participación multisectorial y el respeto a la diversidad cultural, sobre las cuales no se redundará. Sin embargo, cualquiera que sea el modelo a implementar, es imprescindible preguntarnos ¿de qué manera las instituciones, las comunidades y en general, la sociedad, puede brindar acompañamiento y opciones alternativas al suicido en un momento de crisis?

Para responder a esta pregunta se pueden identificar tres grandes conclusiones de este trabajo. En primer lugar, articular modelos interdisciplinarios y multisectoriales de trabajo, lo cual implica una revisión de la vinculación que ha prevalecido entre las instituciones y comunidad, a fin de brindar a las personas y los grupos organizados una participación activa en la identificación de su problemática y la forma de resolver la situación. Las comunidades suelen tener estrategias que han venido utilizando para lidiar con la situación, máxime cuando el suicido se ha convertido en un evento de su vida cotidiana, así que se deben validar y fortalecer las que han mostrado algún nivel de éxito. Una segunda conclusión es que los proyectos particulares deben sostenerse en pilares de puertas abiertas, abordajes grupales que despatologicen la crisis suicida, propicien la escucha y fortalezcan los vínculos, así como una atención oportuna sin la interferencia de requisitos administrativos. Finalmente, y muy vinculado a lo anterior, más allá de los programas estructurados e institucionalizados, se debe buscar la habilitación de espacios de escucha (profesional y no profesional), incluyendo algunos mecanismos como la expresión a través 
del arte. Aunado a esto, se deben orientar los esfuerzos a la promoción de vínculos cálidos y de calidad como una forma de sostén de la vida humana.

¿Cómo hacer esto? Más que pensar en modelos estructurados deben utilizarse los principios de la psicología comunitaria que apuntan a la participación social y a establecer estrategias creativas que involucren a muy diversos actores sociales y a su vez garanticen un adecuado seguimiento.

\section{Referencias}

Castillo Echeverría, Carolina y Maroto Vargas, Adriana. (2014). Diagnóstico comunitario en Dota: el abordaje psicosocial del suicido y el enfoque de salud comunitaria. San José: Universidad de Costa Rica.

Chávez Hernández, Ana María, Medina Núñez, Miriam Carolina y Macías García, Luis Fernando. (2008). Modelo psicoeducativo para la prevención del suicidio en jóvenes. Salud Mental, 31(3), 197-203.

Corpas Nogales, José Manuel. (2011). Aproximación social y cultural al fenómeno del suicidio. Comunidades étnicas amerindias. Gazeta de Antropología, 27(2), 1-17.

Dobles Oropeza, Ignacio. (En prensa). Delimitación disciplinaria y poder: algunas discusiones necesarias en el campo de la psicología comunitaria. En I. Dobles, A. Maroto, MJ Masís y A. Rodríguez. (Ed.), Miradas sentidas y situadas: experiencias con grupos y comunidades. San José: Universidad de Costa Rica.

Escalante Meza, Jimena. (En prensa). Procesos comunitarios, identidad colectiva, diferenciación y territorio en relación con el riesgo "natural”. En I. Dobles, A. Maroto, MJ Masís y A. Rodríguez. (Ed.), Miradas sentidas y situadas: experiencias con grupos y comunidades. San José: Universidad de Costa Rica.

Gonzales-Portillo, Juan, Gil-Arévalo, Jerson, Hernández-Botero, Daniela y Henao-Sánchez, Lina Marcela. (2016). Evaluación de las expectativas negativas y tipo de riesgo suicida en estudiantes de $9^{\circ}, 10^{\circ}$ y $11^{\circ}$ de una institución educativa del departamento del Quindío. Duazary, 13(1), 7-14. doi: http://dx.doi.org/10.21676/2389783X.1582

González-Forteza, Catalina, Arana Quezadas, Daniela Shaíl y Jiménez Tapia, José Alberto. (2008). Problemática suicida en adolescentes y el contexto escolar: Vinculación autogestiva con los servicios de salud mental. Salud Mental, 31(1), 23-27.

Guibert Reyes, Wilfredo. (2002). La promoción de salud ante el suicidio. Revista Cubana de Medicina General Integrada, 18(1), 33-45.

Maroto Vargas, Adriana. (2015). Estado del arte de investigación en suicidio en Costa Rica 1998-2013 (Sin publicar). San José: Universidad de Costa Rica.

Martínez, Carlos. (2008). El abordaje comunitario de la crisis suicida en sus diferentes ámbitos de intervención. Revista Asociación Levantina de Ayuda e Investigación de los Trastornos de la Personalidad, (4). Recuperado a partir de http://suicidologia.info/web/wp-content/uploads/2014/02/ abordajecomunitario.pdf

Mosquera, Laura. (2016). Conducta suicida en la infancia: Una revisión crítica. Revista de Psicología Clínica con Niños y Adolescentes, 3(1), 9-18.

Organización Mundial de la Salud. (2016). Salud mental: fortalecer nuestra respuesta. Recuperado a partir de http://www.who.int/mediacentre/factsheets/fs220/es/

Pérez, Andrea. (2014). Muertes silenciadas: Problemática del suicidio en los campesinos de La Unión (Antioquia). Revista Nacional Facultad de Salud Pública, 32(2), 92-102.

Piedrahita, Laura Elvira, Paz, Karla Mayerling y Romero, Ana Maritza. (2012). Estrategia de intervención para la prevención del suicidio en adolescentes: la escuela como contexto. Hacia promoción de la salud, 17(2), 136-148. 
Rodríguez Gómez, Gregorio, Gil Flores, Javier y García Jiménez, Eduardo. (1996). Metodología de la investigación cualitativa. Málaga: Ediciones Aljibe.

UNICEF (Ed.). (2012). Suicidio adolescente en pueblos indigenas: tres estudios de caso. Panamá: UNICEF.

Valadez, Isabel, Amezcua, Raúl, González, Noé, Montes, Rosalba y Vargas, Vania. (2011). Maltrato entre iguales e intento suicida en sujetos adolescentes escolarizados. Revista Latinoamericana de Ciencias Sociales, Niñez y Juventud, 9(2), 783-796.

Van Dijk, Teun. (1999). El análisis crítico del discurso. Anthropos, (186), 23-36. 
